\title{
Medical History Category
}

National Cancer Institute

\section{Source}

National Cancer Institute. Medical History Category. NCI Thesaurus. Code C83018.

A classification of the medical history. 